Brit. J. prev. soc. Med. (1974), 28, 196-202

\title{
Measuring the effectiveness of health care systems*
}

\author{
ALAN WILLIAMS \\ Department of Economics, University of York
}

\section{The Problem and its Setting}

In my mind's eye I have an image of a health care system whose sole function is to ensure that the community it serves derives the maximum net benefit from its existence. The community it serves (its clients) comprises sick people and those who suffer pain, grief, anxiety, etc. because of that sickness, both now and contingently in the future. It does not include as such those whose livelihood depends upon providing the inputs which the health care system requires, except insofar as they are clients in the meaning given above. Moreover, this community wishes its health care system to be run in a manner which reflects the values of the community, despite the fact that the health care system is so large and complex that a great deal of decentralization of resource-allocation decisions is necessary. The basic problem I wish to examine is 'how can we measure the efficiency of such a system?' or, put in more everyday language, "how can we tell whether that health care system is serving the community as well as it could?'

The simple-minded economist's formulation of this problem would be to establish production functions for the various health-affecting activities which the system might embrace and from these estimate marginal social costs, then elicit the preference function of the community for the outputs of the system, and optimize (i.e., maximize the difference between total social benefits and total social costs). At this level of abstraction this way of formulating the problem might be assigned to that class of economic propositions labelled 'true but unhelpful', but because it is both true and fundamental it is nevertheless a useful reference point for the discussion. This framework is developed further by Culyer, Lavers, and Williams (1972).
In practice we have difficulty implementing it as an operational research strategy for several reasons:

(a) Production functions are ill defined owing to our ignorance of the physiological, psychological, and sociological influences affecting the efficacy of therapeutic or supportive activities. Cochrane (1972) is a particularly pungent exponent of this view.

(b) In costing the inputs into these activities we are often unclear at a conceptual level as to the proper realm of discourse (i.e., over what range of considerations we are suboptimizing in an imperfect world) and, even when we are clear on that score, the data at our disposal are frequently inadequate, drawing heavily on financial data from large public agencies and from the well organized markets which happen to exist, with rather poor data on true opportunity costs, especially as felt by the clients themselves. For instance, the simple notion that the client's time is also valuable is slowly gaining explicit recognition in 'scheduling' and 'location' studies in the health care system. It does not always spill over as fully as it should into comparisons of institutional versus domiciliary care, however, with some odd effects on policy choices ..... see, for instance, Wager (1972).

(c) The community's preference function is a will $o$ ' the wisp because we are not sure the community actually has one, or even has a set of concepts and a language in which to discuss what is involved in developing one, not to mention much idea as to who should play what role in that process.

In this paper I shall not discuss the difficulties arising under $(a)$, though we shall have to take note of them. The agenda of business to discuss under (b) is long and fascinating, and is the area of health studies where the economist's role is pre-eminent. But my interests lie in area (c) because I think it is

\footnotetext{
* Presented to an Advanced Seminar on Health Economics at the Centre for Extension Training in Community Medicine, London School of Hygiene and Tropical Medicine, 1-3 November 1973.

This paper will also appear in The Economics of Health and Medical Care: Proceedings of a Conference held by the International Economic Association at Tokyo, edited by Mark Perlman. Macmillan (1974).
} 
here that the most appalling weaknesses of current studies into the efficiency of health care systems (by economists and others) are manifest, and, although it is not, strictly speaking, economics, there appears to be some reluctance by other professional groups to plunge into the difficult territory of devising output measures for health care systems which are going to serve the economist's purposes in answering the basic question posed earlier.

I therefore begin by setting out below certain desiderata which I think any such output measures should fulfil before going on to outline a strategy for devising such measures.

\section{Desiderata}

Ideally we seek an estimate of the benefits of health-affecting activities measured in monetary units commensurate with the relevant cost estimates. Before setting out the schema by which such measures might be evolved, two cautionary disclaimers are in order: (a) using monetary units as a measure of value does not imply accepting any particular mode of valuation and, more specifically, it does not imply acceptance of market values, and still less of a cash flow approach to the evaluation of health care systems; $(b)$ in order to purge the subsequent discussion of any risk of misunderstanding on that score I am going to use the convention that all the so-called economic benefits (like getting people back to work more quickly, saving costs which would fall on other services, etc.) are treated as negative costs and offset against the items on the input side. This obviously leaves the calculation of net benefits unaffected, but means that we might have to think of some activities as having negative net costs if, for instance, the economic benefits outweighed the costs. Such cases I do not regard as very interesting from our standpoint, since all such activities should obviously be pursued in a rational society, as the humanitarian benefits are free goods. The interesting cases are those where the humanitarian benefits can be obtained only by the sacrifice of other good things (i.e., where their costs are positive). Thus only humanitarian benefits are considered here.

One other important preliminary matter needs to be made clear, and that is that because it is the client's state which is our central interest, the measures we are seeking must be to do with the client's state; this is not as trivial as it seems at first sight. In this context it is important to distinguish between indicators of state-of-health (like mortality and morbidity measures, days of restricted activity, etc.) and provision indicators (hospital beds per thousand population, doctors per thousand, etc.). It is also important not to be led into believing that numbers of cases, episodes of treatment and hospital bed-days occupied are true output measures, even though they refer to clients, because they are concerned with throughput or workload, not with output in terms of amelioration of an individual's state-of-health compared with what it would otherwise have been. A still more tempting trap for the unwary is set by measures of need, such as nursing dependency scales, which indicate the quantity and quality of care required by a patient. Although these measures are closely associated with client state in terms of social functioning, they specify inputs needed, which is precisely what we must avoid prejudging. This distinction is clearly drawn by Carstairs and Morrison (1971).

Moreover, it is the community's valuations of that state which are relevant. This may or may not coincide with the individual's own valuations of his own state, or indeed with any particular individual's valuations of any other client's state. I am going to duck the very large and difficult set of intellectual philosophical problems which surround the processes by which community values emerge. I shall merely refer to a dialogue between the parties, without investigating the power-and-influence relationships at work therein. What I shall do instead is to indicate the proper professional roles of the various parties, and try to provide them with a communication system which reduces the area of ignorance and risks of confusion in the conduct of that dialogue.

This measure of benefits should also be capable of wide application and not specific to particular conditions, or to the inputs of particular services, otherwise it will be of severely restricted value. This means that it has to refer to a context in which the client's state can be assessed in a fairly general manner, and it leads inexorably to an approach based on the general social functioning of the client, i.e., his or her ability to conduct the essentials of everyday living free of pain. This goes well beyond measurement of blood pressure, pulse rate, etc., and also beyond the identification of conditions (e.g., pneumonia, pregnancy, arthritis), and even beyond the noting of physical disabilities (missing index finger, blind in one eye, loss of one leg below the knee) because it rests on the question: 'to what extent is this individual unable to carry out painlessly the activities which the community would expect individuals of that age and sex normally to be able to carry out?'

The final desideratum is that the measure should be capable of being made operational, which implies that it must be capable of routine application and 
use, which in turn implies that it must not make inordinate intellectual demands nor intolerable demands in time and energy upon those responsible for collecting and processing the information. Although high-powered research and development work may be required to test it out in the initial phase, it should be designed to run on low-powered inputs in the long run.

\section{A Solution in Principle}

The basic elements in the proposed scheme are:

(a) a set of descriptive categories concerned with the client's state in terms of pain-free social functioning;

(b) a relative evaluation process that converts these states into index points;

(c) an absolute valuation of points in money terms.

In this section the general properties of each element will be described before presenting a brief account of some empirical work designed to help us with element $(a)$.

If we are to build up an index of health (or, in this case, of ill-health) we need to measure both intensity and duration. Intensity is here interpreted as having two dimensions, pain and restriction of activity. The first step would therefore be to experiment with simple standardized descriptions of painfulness and of the extent to which activity is restricted to see if there is any concensus among medical personnel as to how painful and how restricting particular conditions are, using these descriptive categories. The initial descriptive stagemay be represented as in Figure 1.

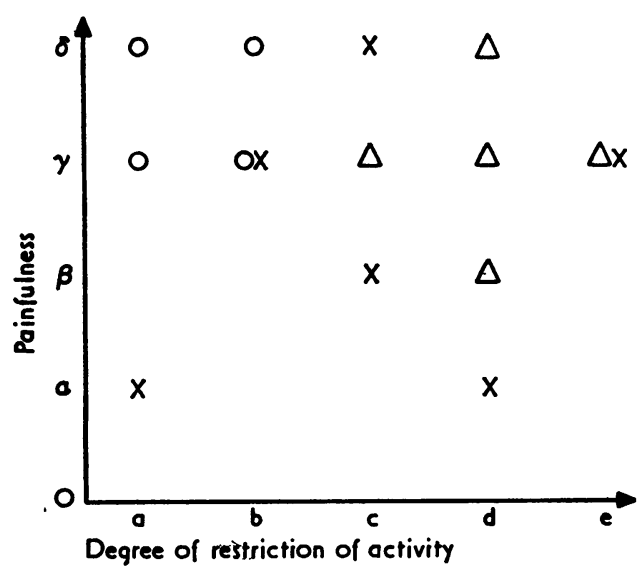

Fio. 1. Simple descriptive statements. $\alpha, \beta, \gamma$, and $\delta$ are simple descriptive statements concerned with painfulness (such as 'mildly uncomfortable', 'very comfortable', 'extremely painful', etc.); a, b, c, d, and e are simple descriptive statements concerned with restriction of activity (such as light work only, confined to house and immediate vicinity, confined to house, confined to bedroom, confined to bed, etc.). The symbols $0, x$, and $\Delta$ each refers to different medical conditions. Each 0 plotted on Fig. 1 represents a different expert's assessment of the most appropriate description of the effect of that condition (e.g., one says $a, \delta$; another says $a, \gamma$; another says $b, \delta$ and yet another $b, \gamma)$. Similarly, each $x$ and each $\Delta$ represent corresponding judgments by various experts of the most appropriate descriptions of each of those conditions. If there is any consistency in these judgments (as there is to $o$ and $\Delta$ in the example), some norm will be indicated as the standard description for that condition; where no concensus exists (as with $x$ in the example), it is likely that the condition under study needs to be more closely specified.

However, supposing that we had each condition clearly ascribed to a pain category $(\alpha, \beta, \gamma$, etc.) and a restricted-activity category (a, b, c, etc.), we would now need to establish the trade-off between them (e.g., is the combination ya better or worse than the combination $\beta c$ ?). This pairwise comparison is essentially a social judgment and should be recognized as such but may have to be made in practice by medical people. This first evaluative step is set out diagrammatically in Fig. 2 where the lines are embryonic indifference curves, and it is desirable to be as 'close' to zero as possible. In the example shown, the combinations $(\beta, O)$, $(\alpha, a)$, and $(0, b)$ are equivalent to each other, but better than $(\gamma, 0)$ and $(0, c)$ (which are equivalent to each other). Those in turn are better than the next group of equivalents $(\beta, a),(\alpha, b),(O, d)$, and so on.

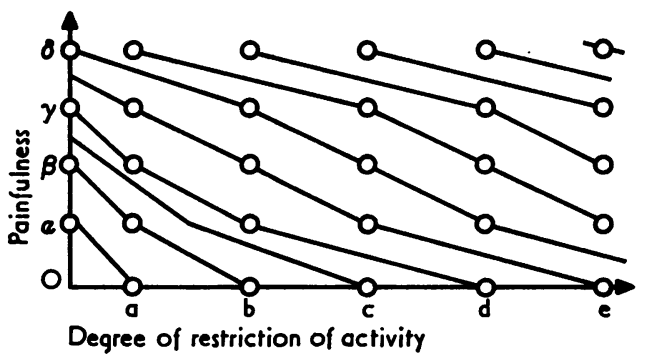

FIG. 2 
Despite the fact that describing the intensity of pain is notoriously difficult, and that interpersonal comparisons are bound to be rather arbitrary, medical personnel can and do make such comparisons between stages and classes of condition, and such comparisons already have to be assimilated into judgments about 'acceptable' degrees of physical disability and pain at the diagnostic and therapeutic level when determining courses of treatment. It is therefore suggested that it should be possible to move to the second (partial evaluative) stage and construct (say) a 10-point scale of intensity of ill-health along the following lines:

$$
\begin{aligned}
0= & \text { normal } \\
1= & \text { able to carry out normal activities, but } \\
& \text { with some pain or discomfort } \\
2= & \text { restricted to light activities only, but with } \\
& \text { little pain or discomfort } \\
3-7= & \text { various intermediate categories reflecting } \\
& \text { various degrees of pain and/or restriction } \\
& \text { of activity } \\
8= & \text { conscious, but in great pain, and activity } \\
& \text { severely restricted } \\
9= & \text { unconscious } \\
10= & \text { dead }
\end{aligned}
$$

Since it is intended to use these numbers as weights, and not simply as rankings, it is important to stress that society's judgments concerning the relative importance of avoiding one state rather than another are represented by the actual numbers attached to each respectively, e.g., state 2 is twice as bad as state 1 , and state 10 is 10 times as bad. This implication must not be shirked, and it must be regarded as a statement about health policy (and is to be made by whoever is entrusted with that responsibility, e.g., the politicians) and not a technical statement about medical condition. In terms of Fig. 2 this would be represented by attaching index numbers to each of the contour lines.

As to duration, this will be based on the outcome of scientific investigations, cast in statistical terms. For instance, recovery from a particular disease will follow one time path (incorporating both intensity and duration) in $90 \%$ of the cases, another in a further $9 \%$, and yet another in the remaining $1 \%$. Chronic cases where no (or little) improvement in intensity is to be expected will have a duration equal to the life-expectancy of that class of individual, and the duration of the gain from postponing death where successful treatment is possible will be similarly measured. A successful treatment is not only one which reduces intensity and duration but could also be one that reduces intensity without affecting duration, or vice-versa; or even that increases one at the expense of the other, providing the net outcome is to reduce the index number (a product of intensity and duration). The important sources of information here are the medical statisticians since it is purely empirical information that is required at this stage in the process.

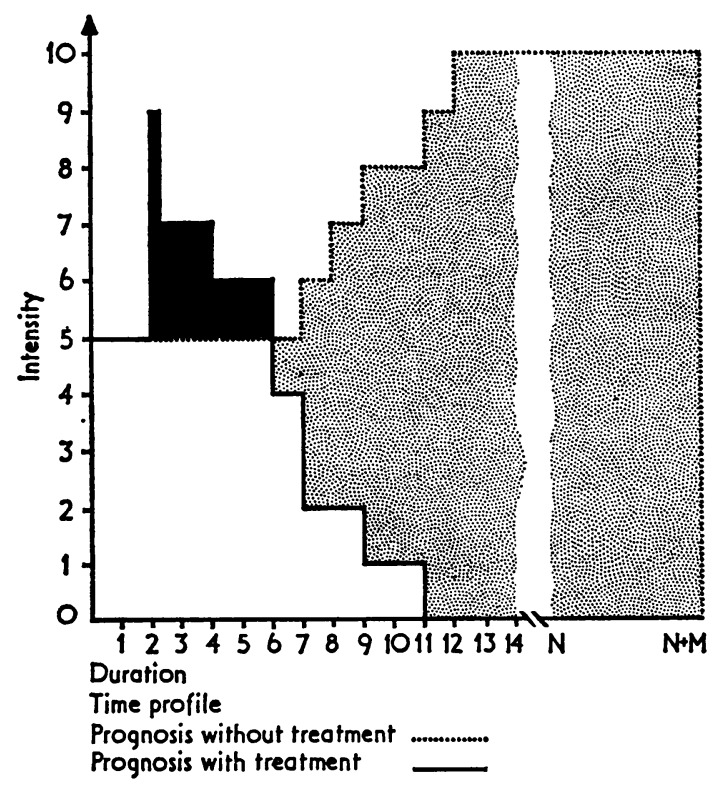

FiG. 3

Figure 3 illustrates how this would work for any particular condition. The diagram starts at a point of time $\mathbf{0}$ when the condition in question is diagnosed. In the illustrative example the first two weeks are spent in further observation, decision as to appropriate treatment, and waiting for therapeutic facilities to become available. The prognosis without treatment (or with the best treatment other than that under consideration) is represented by the broken line (- .-.-), and may be described as a steady deterioration from approximately week 7 until death in week 12. This would be the standard prediction for this class of case. The average expectation of life for a person of that age/sex, etc., is represented as $(n+m)$ which may be rather large if necessary (e.g., 50 years). The prognosis with treatment is represented by the solid line (-), and may be described as two weeks of severe restriction of activity (in the preoperative, operative, 
and immediate postoperative phases) plus possibly considerable pain, with a steady improvement in condition during the ensuing three weeks, a convalescent phase from weeks 7 to 9 , and a further two weeks taking it easy in the normal environment, after which the patient is completely normal (as far as this condition is concerned).

The index score (representing the effectiveness of this treatment) would be the hatched area minus the black area, obviously including in the former the interval omitted in the horizontal scale as drawn. This particular example would obviously be a highly effective treatment if applied to people with long life-expectancy, less so the shorter life expectancy. Both the time profiles used would be derived from statistical analyses of clinical results, on experimental data if the former were lacking. It is up to the medical statistician to provide the key data. A further sophistication which could be introduced if necessary would be to apply a discounting factor which would give less weight to future states of health compared with present states, and hence reflect the greater weight people seem to attach to the 'here and now' rather than to more distant prospects.

Certain features of this system are noteworthy:

(a) it enables preventive as well as therapeutic activities to be incorporated;

(b) although much more difficult in practice, in principle it can embrace mental illness;

(c) it treats one week of suffering at any particular intensity level as being equally undesirable irrespective of the identity of the patient. Other distributional assumptions are possible in principle but would make the analysis much more complicated;

(d) it relates only to patients, and does not include infectivity, or the pain and suffering caused to others by the patient's condition. Neither of these shortcomings is insuperable in principle, but as a practical matter they will be difficult to overcome in the near future;

(e) the satisfaction felt by patients themselves (or their friends and relatives) is not regarded as an independent consideration in this formulation, and if it were it would raise such enormous difficulties for any health indicator that the matter is mentioned here only so that it is not lost sight of.

The primary purpose of such an indicator is to facilitate cost-effectiveness studies by providing a quantification of the purely humanitarian benefits to be used in conjunction with economic costs (and benefits) in order to improve the effectiveness of health services in the face of severe resource limitations. But it could also generate, as a byproduct, improved indicators of the state of (ill) health of a community if used as part of the basic information matrix in a national survey of the state of health of the community. If successful, this would fill an important gap in our present knowledge, for it would include cases where people had not presented themselves for treatment, or where those giving treatment were unaware of the patient's condition between episodes of treatment.

This leaves us with the final step, which is attaching money values to index points. There are various ways of approaching this thorny issue. The one least likely to raise strong emotional objections is simply to calculate the implied vaiues placed on marginal points by existing allocations of resources, and to argue that if there are discrepancies, and the relative points values are right, then resources should be shifted from activities yielding high cost points at the margin to those where marginal points are relatively cheap. A more direct method has been used in the field of crime, by getting respondents of various kinds to say how serious they thought one crime was relative to another, from which an index of crime seriousness was constructed (Sellin and Wolfgang, 1964; Chambers, 1973). Once people get used to this kind of discourse one could then pose the question, 'are these marginal points values of the right order of magnitude relative to other things?' It might help to inform such judgments better by pointing out the kinds of awards which the community regards as fair if someone is moved from health state to health state for a given duration through no fault of his own and to compare this with the sums the community seems willing to devote to preventing similar changes from happening by the provision of health care. Some preliminary analysis of this kind has been done for the UK by Rosser and Watts (1971), and it is likely that more will be undertaken as a result of the recent setting up of a Royal Commission on Civil Liability and Compensation for Personal Injury. Before long we may even reach a stage when some bold politician will have the nerve to put the issue to his constituents in these terms ..... e.g., if elected, my policy will be based on a $5 \%$ increase in the value of a health point (i.e., I think we ought to be prepared to devote an extra $5 \%$ of the community's resources to improving health by one unit at the margins). This price thus becomes a cut-off point for decentralized resource allocation decisions in health care systems and one which will force decision makers to pay 
regard both to medical effectiveness (expected points score) and social valuations (as indicated by the price of a point).

\section{Where Should We Start?}

I am not so foolish as to imagine that by this time next year (or even in 10 years' time) we could have the provision of health care rationalized in this way, even if all the parties whose cooperation is necessary were sold on the idea, so we need to face the problem of research priorities and testing during the developmental learning phase. I am convinced that the major stumbling block at present is the absence of any widely used standardized descriptive categories of social functioning (there are plenty of special-purpose ones, e.g., Hamilton (1960), Isaacs and Walkley (1963), Katz et al. (1963), Brandon and Gruenberg (1966), Tunstall (1966), Harris (1971), Garrard and Bennett (1971)), and without these we cannot get off first base, so I have become a major advocate (and a minor organizer) of longitudinal surveys of clients, these surveys needing to be broad in coverage both as regards the clients' conditions and the health care agencies treating them.

For tactical reasons the care of the elderly has been selected as the test area, covering hospitals, general practitioners, welfare homes, domiciliary support, etc., in both urban and rural areas of England and Wales. In order not to try to clear too many hurdles at once no index is being calculated, so we are operating at the level of Fig. 1 above. This does not, however, preclude individual agencies from supplying their own relative valuations of client states if they wish, and it will give them a set of categories across which they can sensibly do so. Nor does it preclude statistical analysis of the results designed to see what transitions from state to state are associated (ceteris paribus) with the various treatment modes, so that one can begin to build up the basis for prognoses along the lines of Fig. 3, though in several (incommensurable) dimensions rather than in the common currency of 'health points'.

In our study client state has three dimensions (physical mobility; capacity for self-care; and mental state), each of which is ranked on a fourpoint scale, so that there are $4^{3}(=64)$ logically possible different states. Clients are to be reassessed at 3-month intervals so that (if we add the state dead to those already mentioned) it will be possible to compile a $64 \times 65$ matrix of transitions, which can be repeated (and combined) at 3-month intervals to give 3-month, 6-month, 9-month, 12-month, etc. transitions. The fundamental research task will be to try to identify (from a sizeable package of background information on clients, which is also being collected at each assessment) what statistically significant associations exist between the different transitions experienced by people starting in the same state and the different treatment modes provided by the health care system. But a more inimediately important operational pay-off may well be the focussing of routine records on client states and a more general dissemination and testing of category systems of this kind (see Appendix for a brief outline of the contents of schedule to be used).

This project will be at the pilot stage (with some 500 test observations) during 1974, and if no fatal flaws emerge, will run on a fairly large scale $(10,000$ or so observations) during 1975 and 1976. But it will still be but a beginning in the task ahead, because it still will not include any agreed set of index weights across client-states (still less their evaluation in money terms), nor will it provide detailed knowledge of production functions but only broad indicators of the relative effectiveness for one client group of whole packages of care, and it is not concerned with the measurement of costs. However, it is focussed on the appropriate primal element in the problem, and is stage one of a carefully mapped escape route from the intellectually imprisoning confines of the measures of workload and throughput (and even input) which too often are pressed into service as measures of the effectiveness of health care systems.

\section{REFERENCES}

Brandon, S. and Gruenberg, E. M. (1966). The measurement of the incidence of chronic severe social breakdown syndrome. Milbank mem. Fd Quart., 44, Suppl. 129.

CARSTAIrs, Vera and Morrison, M. (1971). The Elderly in Residential Care. Report of a survey of homes and their residents. Scottish Home and Health Department. [Scottish Health Service Studies No. 19.]

Chambers, M. L. (1973). Construction of a utility function for a police force. Paper presented to a RES Specialist Conference on Decision Analysis, Lancaster, January 1973.

Cochrane, A. L. (1972). Effectiveness and Efficiency: Random Reflections on Health Services. Nuffield Provincial Hospitals Trust, London.

Culyer, A. J., Lavers, R. J., and Williams, A. (1972). Health indicators. In Social Indicators and Social Policy, edited by A. Shonfield and Stella Shaw, p. 94. Published for the Social Science Research Council, by Heinemann, London.

GARRARD, Jessie and BenNETt, A. E. (1971). A validated interview schedule for use in population surveys of chronic disease and disability. Brit. J. prev. soc. Med., 25, 97. 
Hamilton, M. (1960). A rating scale for depression. J. Neurol. Psychiat., 23, 56.

HARRIS, A. I. (1971). Handicapped and Impaired in Great Britain, HMSO, London.

IsaAcs, B. and WalkLey, F. A. (1963). The assessment of the mental state of elderly hospital patients using a simple questionnaire. Amer. J. Psychiat., 120, 173.

Katz, S., Ford, A. B., Moskowitz, R. W., Jackson, B. A., and JAFFe, M. W. (1963). Studies of illness in the aged. The index of ADL: a standardized measure of biological and psychosocial function. J. Amer. med. Ass., 185, 914.

Rosser, RAchel and WatTs, V. (1971). The measurement of hospital output. Paper presented to the Operational Research Society Conference, Lancaster, September 1971.

Sellin, T. and Wolfgang, M. E. (1964). The Measurement of Delinquency. Wiley, New York: and London.

TUNSTALL, J. (1966). Old and Alone. A Sociological Study of Old People. Routledge and Kegan Paul, London.

WAGER, R. (1972). The Care of the Elderly: an Exercise in Cost Benefit Analysis commissioned by Essex County Council. Chartered Institute of Public Finance and Accountancy, London.

\section{APPENDIX}

Outline Contents of Assessment Schedule and BaCkGROUND INFORMATION CONCERNING CARE OF THE ELDERLY

\section{A. Assessment SChedule}

The assessment schedule has three divisions concerned with mobility, capacity for self-care, and mental state. The factors considered in each of the divisions are as follows:

1. Mobility

(a) Ability to get in and out of bed and/or chair

(b) Ability to negotiate a level surface

(c) Ability to climb stairs

(d) Ability to walk outdoors

2. Capacity for self-care

(a) Ability to feed self

(b) Ability to dress self

(c) Ability to wash self

(d) Ability to make a hot drink

(e) Ability to cook a meal

(f) Ability to light a fire

(g) Ability to shop

(h) Whether or not continent

3. Mental state

(a) Intellectual processes-memory and orientation of person and place

(b) Loneliness and desolation (c) Depression

(d) Boredom

(e) Motivation towards independence

(f) Anxiety

( $g$ ) Antisocial or self-harming behaviour

Care has to be taken in this division to distinguish general feelings of mood from pathological mental disorders which are to be recorded in the background information.

B. BACKGROUND INFORMATION

1. Personal and socio-economic status

(a) Age

(b) Sex

(c) Marital status

(d) Occupation or previous occupation of self or spouse

(e) Income

2. Domestic environment

(a) Type of house

(b) Facilities, e.g., hot water, location of toilet, bath, etc.

(c) Shared or sole use of accommodation

(d) Warden or other people officially in attendance

(e) Presence of alarm system

(f) Residential or nursing home-public or private -type of home -facilities available

(g) Hospital ward -general, medical, or surgical

-geriatric-assessment or rehabilitative -continuing care -psychiatric

3. Medical condition

Based on broad systemic classes, e.g., Injuries or diseases of: Cardiovascular system Locomotor system Central nervous system

4. Social contacts

(a) Visits received from relatives friends and neighbours

(b) Visits made to tradesmen, etc.

(c) Casual visits to relatives friends and neighbours social clubs shops church, etc.

(d) Other 'non face-to-face' contacts, e.g., telephone calls letters

(e) Interest in current affairs-newspapers, radio, TV

(f) Use of spare time

\section{Use of services}

Use of local authority, hospital, general practitioner, etc., and voluntary services. 